\title{
Spanien als Exil- und Transitland
}

\author{
Patrik von zur Mühlen
}

Spanien ist als Asyl für politisch und rassisch Verfolgte aus dem nationalsozialistischen Deutschland bislang wenig beachtet worden. Die Ereignisse des Spanischen Bürgerkrieges haben das Interesse an spanischer Geschichte so sehr in Anspruch genommen, daß die Bedeutung des iberischen Landes für die Fluchtund Emigrationsbewegung kaum zur Kenntnis genommen wurde. ${ }^{1}$ Dabei hat Spanien sowohl vor Beginn als auch nach Ende des Bürgerkrieges für die deutsche Emigration eine wichtige Rolle gespielt, zunächst als Refugium für Verfolgte, später als Fluchtweg, als zeitweilig einzige Transitstrecke, die aus dem nationalsozialistisch beherrschten Europa in Sicherheit führte.

Für den ersten der hier angesprochenen Zeitabschnitte, also zwischen 1933 und 1936, ist freilich festzustellen, daß Spanien rein zahlenmäßig niemals den Rang von Aufnahmeländern wie Frankreich, den Niederlanden, der Tschechoslowakei, der Sowjetunion oder der USA erlangte. Dennoch bildete es für einige Tausend Flüchtlinge aus Mitteleuropa ein relativ sicheres Terrain, aus dem sie erst durch den Franco-Putsch im Juli 1936 gewaltsam vertrieben wurden. Denn trotz der politischen Instabilität in Spanien vor dem Bürgerkrieg, trotz der Armut und Rückständigkeit des Landes bot es vor anderen europäischen Ländern doch einige beachtliche Vorteile. Man konnte ohne bürokratische Schwierigkeiten einreisen und benötigte keine Aufenthaltsgenehmigung und fast noch wichtiger - keine Arbeitserlaubnis. Spanien war für viele HitlerGegner, die in Frankreich oder anderen Asylländern Schwierigkeiten zahlreicher und vielfältiger Art hatten, so etwas wie ein "Geheimtip". Den Vorteilen des spanischen Exils standen freilich auch Schattenseiten gegenüber. Der Arbeitsmarkt bot nur wenige Chancen, das Einkommen war gering, soziale Sicherheiten fehlten völlig. Es sind etliche Fälle von Emigranten bekannt, die mittellos durch das Land streunten und teilweise wegen Landstreicherei von den spanischen Behörden eingesperrt wurden (Mühlen 1985, 42ff.).

Man kann die nach meiner Schätzung höchstens etwa 5.000 Personen umfassende deutschsprachige Emigration in Spanien bei fließenden Grenzen in drei unterschiedliche Kategorien einteilen: 1) jüdische Emigranten, 2) politische Emigranten und 3) eine schwer zu beschreibende Kategorie, die alle übrigen möglichen Gruppen umfaßt. Die Anfänge der jüdischen Emigration reichen teilweise in die Zeit vor Hitlers Machtergreifung zurück und haben daher nicht in allen Fällen unmittelbar mit ihr zu tun. Nach dem Ersten Weltkrieg ereignete sich in der spanischen Öffentlichkeit die Wiederentdeckung der Spaniolen, also

${ }^{1}$ Mühlen 1985, 39-44; Hermsdorf et.al. 1981, 191-201; ausführlicher wird das Thema behandelt in Mühlen 1992 . 
der in den Balkanländern und im östlichen Mittelmeerraum beheimateten spanischsprachigen sephardischen Juden, deren Vorfahren im 16. Jahrhundert von der Inquisition außer Landes gejagt worden waren. Namhafte Intellektuelle forderten die Rückkehr der verlorenen Kinder Spaniens, um dadurch die vierhundert Jahre zuvor verübte Schandtat der Vertreibung zu annullieren. 1924 hatte ein königliches Dekret den Spaniolen die Möglichkeit zur Wiedererlangung der spanischen Staatsbürgerschaft eröffnet. Die 1931 ausgerufene Republik setzte diese judenfreundliche Politik fort, was wiederum die jüdische Hilfsorganisation HICEM dazu veranlaßte, mit der Regierung über die Ansiedlung jüdischer Einwanderer zu verhandeln. Tatsächlich ließen sich einige Tausend Juden meist russischer und polnischer Herkunft in Spanien nieder. 1933 folgten ihnen in verstärktem Maße Juden aus Deutschland. Genauere Zahlen liegen nicht vor, jedoch bewegen sich Schätzungen bei 2.000 bis 3.000 Personen für Barcelona und etwa weiteren 1.000 bis 2.000 für das übrige Spanien. ${ }^{2}$

Bei den politischen Emigranten aus Deutschland handelte es sich vornehmlich um Kommunisten, von denen etwa 40 bis 60 für Barcelona und 200 für ganz Spanien geschätzt werden ${ }^{3}$, zu denen man noch ein milieuspezifisches Umfeld von Familienangehörigen und politisch nicht organisierten Gesinnungsfreunden hinzurechnen kann. Dazu kamen einige Dutzend Anarchosyndikalisten und vereinzelte Sozialdemokraten sowie Angehörige anderer linker Parteien und Gruppierungen. Sie fanden in den spanischen Schwesterorganisationen Unterstützung, zum Teil auch Arbeit, und bildeten in den großen Städten, von denen an erster Stelle Barcelona zu nennen ist, ein eigenes Emigrantenmilieu. Ab Juni 1933 gab ein gewisser Ludwig Stautz in der katalanischen Hauptstadt ein vierzehntägig erscheinendes linkes, aber parteipolitisch nicht festgelegtes Blatt, Der Antifaschist, in deutscher und spanischer Sprache heraus, das dann im November desselben Jahres vermutlich infolge eines amtlichen Verbots sein Erscheinen einstellen mußte.

Die dritte Gruppe umfaßte Intellektuelle aller Art und Richtungen, Publizisten, Schriftsteller, Maler, Photographen, - kurz Künstler, auch Lebenskünstler und das, was man heute eine "alternative Szene" nennen würde. Walter Benjamin lebte 1933 ein halbes Jahr auf den Balearen, ebenso der Verleger und Publizist Harry Graf Kessler und die Journalisten Alexander Einbinder und Franz v. Puttkamer, die Schriftsteller Albert Vigoleis Thelen, Erich Arendt, Heinz Kraschutzki und Franz Blei, die Maler Alfred Otto Wolfgang Schulz ("Wols") und Arthur Segal, namhafte Photographen wie Hans Namuth, Georg Reisner, Walter Reuter und viele andere. ${ }^{4}$ Auch zu dieser in sich sehr heterogenen Kategorie muß noch ein Umfeld von Freunden, Gleichgesinnten und Schicksalsgenossen hinzugerechnet werden.

\footnotetext{
${ }^{2}$ Vgl. Tartakower/Grossmann 1944, 313; Ysart 1973, $28 \mathrm{f}$.

${ }^{3}$ Hermsdorf et.al. 1981, 200; Mühlen 1985, 41.

${ }^{4} \mathrm{Vgl}$. Mühlen 1985, 39; Hermsdorf et.al. 1981, $191 \mathrm{ff}$.
} 
Blickt man auf die geographische Verteilung der deutschen Emigranten innerhalb Spaniens, dann zeigen sich deutliche Schwerpunkte. Das wichtigste urbane Zentrum der jüdischen, politischen und intellektuellen Emigration war Barcelona, in wesentlich geringerem Maße Madrid. Ein weiteres Zentrum lag auf den Balearen, vor allem Mallorca. Nur relativ kleine Gruppen verteilten sich auf die auch damals schon frequentierten Badeorte der Mittelmeerküste und sogar auf die Kanarischen Inseln. Die meisten dieser Emigranten wurden durch den Franco-Putsch überrascht und nach ihrer Emigration aus Deutschland erneut aus ihrer bisherigen Lebensbahn geworfen. Von den politischen Emigranten beteiligten sich viele an den Abwehrkämpfen gegen den Franco-Putsch, reihten sich in die antifaschistischen Milizen und später in die Internationalen Brigaden ein. Andere verließen fluchtartig Spanien. Einige wurden von der Falange oder vom Militär festgenommen und für viele Jahre eingesperrt. ${ }^{5}$

Mit dem Ausbruch, spätestens jedoch mit dem Ende des Bürgerkrieges, den ich hier überspringe, mußten die meisten Emigranten Spanien in Richtung Frankreich verlassen. Eine nicht näher festzustellende Zahl geriet in die Gefangenschaft der Franco-Truppen und wurde interniert, vereinzelt wohl auch der Gestapo ausgeliefert. Auch die überwiegend jüdische Emigration hatte 1936 Spanien verlassen. Nur wenige Familien - etwa zwei oder drei Dutzend Personen - blieben unter der Franco-Herrschaft im Lande, schikaniert und eingeschüchtert durch die antijüdische Hetze der Falange (Wasserstein 1979, 236). Aber Spanien nahm jetzt - im Jahre 1939 - ohne eigenes Zutun eine neue Rolle für die Emigration aus dem deutschen Machtbereich an. Mit dem Kriegsausbruch im September 1939 war ein Teil Europas de facto für die Auswanderung gesperrt. Es gab zunächst eine gewisse Emigration nach Ostasien auf dem Landwege durch die Sowjetunion, über die Balkanländer in den Nahen Osten und über Italien nach Übersee. Aber nachdem Mussolini im Juni 1940 auf deutscher Seite in den Krieg eingetreten war und Hitler und seine Verbündeten im April 1941 Jugoslawien und im Juni die Sowjetunion überfallen hatten, entfielen diese Länder als mögliche Fluchtwege. Wer aus dem nicht besetzten Teil Frankreichs - und hier konzentrierte sich das Gros der Emigration in Europa - nach Großbritannien oder nach Übersee fliehen wollte, mußte hierzu die iberische Fluchtroute wählen. Dies war aus vielfachen Gründen ein schwieriges Unternehmen, war doch Spanien Mitglied des Antikomintern-Paktes und galt Franco - zu Unrecht, wie wir heute wissen - als enger Verbündeter Hitlers.

Die Rolle Spaniens als Transitland erfordert eine knappe Übersicht über seine Stellung in der internationalen Staatenwelt während des Zweiten Weltkrieges. Gegen Ende des Bürgerkrieges und unmittelbar danach demonstrierte Spanien deutlich seine ideologische Nähe und politische Komplizenschaft mit

${ }^{5}$ Vgl. Lustiger 1989, 49; Brigada Internacional 1976, 51-56. 
Deutschland und Italien. Der Beitritt zum Antikomintern-Pakt und der Austritt aus dem Völkerbund im März bzw. Mai 1939 unterstrichen dies ebenso wie die Besetzung der internationalen Tanger-Zone nach Beginn des Zweiten Weltkrieges. Wirtschaftlich war Spanien von beiden Achsenmächten abhängig, nicht zuletzt wegen der Kriegsschulden für die geleistete Waffenhilfe im Bürgerkrieg. Andererseits brach Franco nicht völlig mit der Gegenseite und erneuerte Handelsbeziehungen mit Großbritannien und den USA (Bernecker 1983, 80f.). In der Außenpolitik verfolgte Madrid das Ziel, sich von Deutschland nicht vereinnahmen und in den Krieg hineinziehen zu lassen. Wiederholt lehnte es das Ansinnen ab, sich durch einen Überfall auf Gibraltar am Kriegsgeschehen zu beteiligen - wie dies übrigens von Kreisen des spanischen Militärs und der Falange gewünscht worden war. Stattdessen wählte Madrid einen Kurs, der über die Stadien einer erklärten "Neutralität", einer "Nicht-Kriegführung", der Entsendung der Blauen Division nach Rußland bis zu einer faktischen Äquidistanz zu den beiden Blöcken mit einer vorsichtigen Verlagerung der Präferenzen von den Achsenmächten hin zu den Westalliierten führte (Detwiler 1962; Hillgruber 1965).

Die deutsche Politik versuchte nun, die innerspanischen Fraktionsbildungen in ihrem Sinne zu nutzen und alle die Kräfte zu stärken, die für einen Überfall auf Gibraltar eintraten, während Kreise der spanischen Wirtschaft und die Anhänger des exilierten Königshauses Bourbon-Parma einen eher probritischen Kurs verfolgten. Mit massiven Mitteln bemühten sich die deutsche Botschaft in Madrid, die NSDAP-Auslandsorganisation, die Abwehr und das Reichssicherheitshauptamt, die spanische Innenpolitik zu beeinflussen (Ruhl 1975, 95-122). Ein Heer von Agenten der genannten Dienststellen - getarnt als Firmenvertreter, Lufthansa-Kapitäne, Gastdozenten, Sprachlehrer, Techniker in der Industrie - und anderen war bemüht, ein Geflecht von pronazistischen Sympathisanten als innenpolitischen Faktor aufzubauen und den Kurs des Landes zu bestimmen. Britische Stellen schätzten die Zahl der im Lande sich aufhaltenden Deutschen im Jahre 1939 auf 80.000 , bei steigender Tendenz während des Krieges. ${ }^{6}$ Dies mag in der Größenordnung zwar maßlos übertrieben sein, deutet aber richtig die starke deutsche Präsenz in Spanien an. Angstvolle Gerüchte unter Flüchtlingen und Emigranten, sie würden sich auf spanischem Boden de facto in den Händen der Gestapo befinden, schienen nach damaligem Kenntnisstand nicht ganz abwegig zu sein. Es gilt daher zu prüfen, wie weit der "lange Arm" der Gestapo tatsächlich reichte und wozu er fähig war.

Vorab aber sollte geklärt werden, wieviele Personen und welche Personengruppen seit Beginn des Zweiten Weltkrieges Spanien durchquerten. Das Interesse meines Beitrages gilt vorrangig der deutschsprachigen Emigration, die

${ }^{6}$ Vgl. Hamilton 1943, 179f.; Hoare 1946, 233, 236, 268. 
aber nur einen Teil der Transit-Emigration ausmachte. Exakte Gesamtzahlen liegen nicht vor und sind wegen der zahlreichen illegalen Grenzübertritte aus archivalischen Quellen wohl auch nicht zu ermitteln. Schätzungen über etwa 50.000 Flüchtlinge zwischen 1939 und Sommer 1944 scheinen mir zu niedrig angesetzt ${ }^{7}$, nicht zuletzt, weil die Schätzungen für die Transit-Emigration über Portugal, die mit der Spanien-Emigration weitgehend identisch war, wesentlich darüberliegen. Die tatsächliche Zahl dürfte wohl irgendwo zwischen 50.000 und 100.000 liegen. Die Mehrheit hiervon bildeten jüdische Flüchtlinge ehemals deutscher, österreichischer und tschechoslowakischer Nationalität, aber auch aus Polen, Litauen und anderen besetzten Ländern Europas, dazu aus Ungarn, Rumänien und Jugoslawien. Eine zahlenmäßig geringere Gruppe machten Angehörige politischer Gruppen im französischen Exil aus, dazu Intellektuelle, Schriftsteller und Künstler. Eine dritte Gruppe bildeten alliierte Luftwaffensoldaten, die von den Deutschen abgeschossen und inhaftiert worden waren, aber aus der Haft hatten entkommen können; sie versuchten, sich über Spanien nach Gibraltar durchzuschlagen. Ein großes Kontingent machten nach der Besetzung Vichy-Frankreichs durch die Deutschen im November 1942 Franzosen aus, die ab Februar 1943 weiteren Zulauf durch vorwiegend jüngere Landsleute erhielten, die sich der Rekrutierung für den Arbeitsdienst in Deutschland entziehen und sich stattdessen in Nordafrika den Forces Françaises Libres anschließen wollten. Als letzte, freilich sehr kleine Gruppe sind deutsche Deserteure zu nennen, die sich kurz vor Abzug der Wehrmacht von der Pyrenäengrenze nach Spanien absetzten. Für jede dieser Gruppen stellten sich die Probleme einer Flucht über die Pyrenäen anders dar.

Wollte man legal nach Spanien ausreisen, benötigte man das französische visa de sortie sowie das portugiesische Transitvisum. Dieses erhielt man wiederum nur, wenn man das Einreisevisum eines Ziellandes und ggf. auch ein Passageticket vorweisen konnte. Der Emigrant mußte also mühselige bürokratische Prozeduren hinter sich bringen, wobei es oftmals geschah, daß er das zuletzt beantragte Dokument erhielt, nachdem das zuerst beantragte seine Gültigkeit verloren hatte und somit die Sisyphusarbeit von vorne begann. Hatte er alle diese Papiere beisammen, konnte er in der Regel ohne Schwierigkeiten Spanien auf einem beliebigen Wege in Richtung Portugal durchqueren. Mir sind in den Quellen keine Fälle begegnet, in denen Flüchtlinge mit ordnungsgemäBen Papieren verhaftet oder an der Ausreise gehindert worden wären. Personen, die sich während des Bürgerkrieges in den Internationalen Brigaden oder sonstwie auf republikanischer Seite engagiert hatten, erhielten, wenn die spanischen Stellen darüber informiert waren, kein Transitvisum. Dennoch sind einige Vertreter linker deutscher Parteien - auch solche, die zeitweilig in Spanien gekämpft hatten - unter ihrem regulären Namen ungehindert durch das Land

${ }^{7}$ Vgl. Gilbert 1969, 87; Bernecker 1983, 82; Ysart 1973, 43. 
gereist: Max Diamant, Fritz Heine, Erich Ollenhauer, Friedrich Stampfer, die früheren Kommunisten Ruth Fischer und Arkadij Maslow und andere. ${ }^{8}$

Das Problem für einen großen Teil der Flüchtlinge aller Nationalitäten bestand nun darin, daß sie eines dieser erforderlichen Dokumente nicht erbringen konnten und daher Spanien illegal betreten mußten. Manche wurden unmittelbar an der Grenze nach Frankreich zurückgeschickt, andere wurden weiter im Landesinnern bei Razzien und Kontrollen gefaßt und verhaftet, wenn sie das Einreisevisum und das damit verbundene salvo conducto nicht vorweisen konnten. Wieder andere fielen auf, weil ihre Papiere zu plump gefälscht waren. Schließlich bildete eine falsch ausgefüllte Devisenerklärung einen der häufigsten Gründe für Festnahmen durch die Spanier. In manchen Fällen, vor allem an der Grenze, konnten stattliche Schmiergelder oder französische Zigaretten eine Verhaftung verhindern.

Wie stark waren nun diese Verhaftungen politisch motiviert und wie weit steckten möglicherweise deutsche Agenten dahinter? Bekanntlich bestand seit Juli 1938, also noch aus Bürgerkriegszeiten, ein zwischen dem spanischen Innenminister und Heinrich Himmler als Chef des Reichssicherheitshauptamtes unterzeichnetes Polizeiabkommen über die gegenseitige Unterstützung bei der "Bekämpfung politischer Verbrecher". Dieses Abkommen enthielt allerdings nur vage Absichtserklärungen und keine Details und war überdies niemals ratifiziert worden. Beide Seiten hielten sich also pragmatisch an das Abkommen, je nachdem wie es ihnen paßte. Unter Berufung auf dieses Abkommen wurden 1939 sogenannte Polizeiattachés mit Diplomatenstatus in der deutschen Botschaft untergebracht, die allesamt Mitarbeiter des Reichssicherheitshauptamtes waren. Ihr Leiter und damit SD-Chef für Spanien war SS-Sturmbannführer Paul Winzer. Die Zahl seiner offiziellen Mitarbeiter war mit 19 Anfang 1941 nicht sehr groß, jedoch muß man die vielen getarnten deutschen Agenten und eine beträchtliche Zahl spanischer Sympathisanten und Mitarbeiter aus Falange, Militär, Staatsapparat und vor allem der Dirección General de Seguridad hinzuzählen (Ruhl 1975, 58). Man weiß, daß in grenznahen Orten zahlreiche Beamte ebenso wie Zivilisten in deutschem Sold standen. Nach unbewiesenen und wohl auch etwas spekulativen Aussagen des Amerikaners Varian Fry, der sich von Marseille aus für die Rettung zahlreicher Flüchtlinge einsetzte, wurde jeder Antrag auf ein spanisches Transitvisum vorher der Gestapo zur Genehmigung vorgelegt. Von Emigranten wird mehrfach belegt, daß in Eisenbahnzügen Männer in spanischen Uniformen patrouillierten und dabei durch ihr unspanisches Aussehen ebenso wie durch ihren deutschen Akzent auffielen. Selbst unter den Emigranten sollen sich getarnte SD-Agenten befunden haben. ${ }^{9}$

${ }^{8}$ Vgl. das Schreiben Fritz Heines vom 18.3.41 an Friedrich Stampfer; Archiv der sozialen Demokratie/Bonn: Nachlaß Friedrich Stampfer II, 38. Vgl. auch Stampfer 1957, 279f.; Fischer/Maslow 1990, 93.

${ }^{9} \mathrm{Vgl}$. Feuchtwanger o.J., 254f.; Ruhl 1975, 57f.; Hamilton 1943, 178f.; Fry 1986, 116-118. 
Kann man hier von einem "langen Arm" der Gestapo sprechen? Es gab einige Entführungsfälle, von denen allerdings nur einer einen Emigranten betraf: Der linke Publizist Berthold Jacob, der 1935 schon einmal von der Gestapo aus Basel nach Deutschland entführt, aber auf massiven Protest der Schweiz wieder freigelassen worden war, floh im April 1941 mit gefälschten Papieren unter dem Namen Marcel Rollin und als angeblicher peruanischer Staatsbürger, der kein Spanisch verstand, bis zur portugiesischen Grenze. Erst dort fiel den spanischen Beamten die Fälschung der Papiere auf. Er wurde verhaftet, zunächst eingesperrt, dann aber unter Polizeiaufsicht in Madrid freigelassen. Mit Hilfe der amerikanischen Unitarier wurde er illegal, aber problemlos nach Portugal geschleust. Jacob war nun aufgrund seiner langen publizistischen Tätigkeit als Mitarbeiter der Neuen Weltbühne und als scharfer Kritiker des Nazi-Regimes sehr gefährdet. Tatsächlich wurde er im Sommer 1941 in Lissabon gekidnappt, nach Spanien gebracht und von dort nach Berlin verschleppt. Er starb im Februar 1944 in Berlin. Seine Entführung kann nur mit Wissen und Duldung, wahrscheinlich mit aktiver Mitarbeit portugiesischer und spanischer Geheimdienststellen erfolgt sein. Es gibt noch einige andere Fälle, in denen ein deutscher Einfluß bei Verhaftungen möglich, aber im einzelnen nicht nachweisbar ist.

Es bestand unter den Emigranten verständlicherweise eine starke Furcht vor dem Transit durch Spanien. Es kursierten Gerüchte, daß die Spanier politisch Verfolgte an die Gestapo ausgeliefert hätten. Derartige Berichte fanden auch Eingang in die Literatur der Kriegs- und frühen Nachkriegszeit und haben wesentlich zu dem düsteren Spanienbild jener Zeit beigetragen. ${ }^{10}$ Sämtliche Hinweise auf spanische Auslieferungen von Flüchtlingen an die Gestapo nennen keine Namen, keine Daten oder sonstige Details und berufen sich selbst nur auf Gerüchte. Hierbei ist folgendes zu unterscheiden. Es gab einige wenige Auslieferungen gefangener Deutscher und Österreicher, die vor 1939 in den Internationalen Brigaden gekämpft hatten. Aber dies ist ein anderer Personenkreis als der, dessen spanischer Fluchtweg hier nachgezeichnet werden soll. Es gab überdies Fälle von Zurückweisung und Abschiebung illegal eingereister Emigranten über die Grenze nach Frankreich. Ein solcher Vorgang mag dann, wenn auf der anderen Seite Vichy-französische oder nach Besetzung ganz Frankreichs deutsche Patrouillen die zurückgewiesene Person festnahmen, verhängnisvolle Konsequenzen gehabt haben. Aber in diesen Fällen handelte es sich sowohl im juristisch-administrativen Sinne als auch der Intention der Spanier nach nicht um eine Auslieferung. Soweit die allerdings lückenhaften Quellen hier eine Aussage zulassen, hat es keine Auslieferungen gegen den Willen der Betroffenen an die Deutschen gegeben. Das gilt für politisch gefährdete Personen ebenso wie für die meist unpolitischen Angehörigen der sog. jüdischen Massenemigration. Wenn also Walter Benjamin sich im katalanischen

${ }^{10} \mathrm{Vgl}$. Abusch 1981, 581; Robinson 1953, 10; Hamilton 1943, 178-180. 
Port Bou mit einer Überdosis Morphium das Leben nahm, weil er eine Verhaftung, Abschiebung oder gar Auslieferung befürchtete, dann handelte es sich um eine psychologisch erklärbare, sachlich aber nicht gerechtfertigte Überreaktion, die auf einer Fehleinschätzung der spanischen Behörden beruhte.

Mehrere Jahre nach dem Kriege schlug das Pendel in der Bewertung Spaniens in die andere Richtung und führte erneut zu Zerrbildern: Das FrancoRegime als selbstloser Retter der bedrohten Juden, Spanien als einziges Land, das den Verfolgten Einlaß und Durchlaß gewährte, während alle anderen Länder ihre Grenzen schlossen (Ysart 1973, 49f.). Auch an dieser Darstellung sind erhebliche Korrekturen anzubringen. Madrid nahm in der Frage der Transit-Emigration eine schwankende Rolle ein, die im übrigen seiner Außenpolitik entsprach. Während die deutsche Seite Spanien drängte, alliierte Soldaten oder junge wehrfähige Franzosen nicht durchzulassen oder gar auszuliefern, übten die angelsächsischen Botschaften Druck in entgegengesetzter Richtung aus, von dem dann auch Emigranten und Flüchtlinge aller Länder profitierten. Als die deutschen Truppen im November 1942 als Antwort auf die britisch-amerikanische Landung in Nordafrika auch Vichy-Frankreich besetzten, kontrollierten sie die gesamte Pyrenäengrenze und versuchten, sie hermetisch abzuriegeln. Eine legale Transit-Emigration gab es nun nicht mehr, wohl aber eine massiv ansteigende illegale Emigration. Aus Furcht vor deutschen Repressalien verkündete Spanien im Februar 1943 seinerseits die generelle Schließung der Pyrenäengrenze und drohte an, alle clandestinos nach Frankreich abzuschieben, was in dieser Zeit in der Tat einer Auslieferung gleichgekommen wäre. Diese Ankündigung löste einen diplomatischen Sturm der Entrüstung aus. Churchill selbst erklärte dem spanischen Botschafter in London, daß das Vereinigte Königreich dies als unfreundlichen Akt betrachten würde. In Madrid protestierten die amerikanische Botschaft und auf ihre Veranlassung auch die Botschaften Portugals und Argentiniens sowie die Apostolische Nuntiatur. Spanien lenkte ein und widerrief die angekündigte Abschiebung, hielt aber bis zum Sommer 1944 seine Grenze zu Frankreich geschlossen ${ }^{11}$. Nach der Befreiung Frankreichs entfielen die Motive für eine Flucht, und der Flüchtlingsstrom hörte schlagartig auf, so daß die Grenzen wieder geöffnet werden konnten.

Was taten nun die spanischen Behörden mit den Personen, die sie wegen eines illegalen Grenzübertritts oder eines Devisenvergehens festgenommen hatten? In der Regel wurden die verhafteten Ausländer für etwa fünf oder sechs, manchmal auch mehr Monate in einem Gefängnis eingesperrt und ohne Gerichtsprozeß festgehalten. Allerdings durften sie Kontakt zu Anwälten aufnehmen oder sich gegen Geld vom Gefängnispersonal zusätzliche Lebensmittel kaufen, um die erbärmliche Gefängniskost aufzubessern. Später wurden sie dann in Konvoys von 40 bis 120 Personen in eines der beiden Internierungslager Miranda de Ebro in Navarra oder Nanclares de la Oca in der benachbarten

\footnotetext{
${ }^{11}$ Wasserstein 1979, 205f., 211f.; Ysart 1973, 49.
} 
baskischen Provinz Alava verlegt. Für verhaftete Franzosen gab es im Baskenland ein eigenes Lager - Uberagua de Ubilla. Diese Lager führten die unmißverständliche Bezeichnung campo, gelegentlich auch depósito de concentración. Trotzdem dürfen sie nicht mit deutschen Konzentrationslagern gleichgesetzt werden. Sie dienten der Internierung, nicht aber der Verfolgung oder gar Vernichtung der Häftlinge. Es gab keinen Stacheldraht, keine elektrischen Zäune, keine Wachtürme oder Minenfelder, sondern nur eine steinerne Mauer, die leicht überwunden werden konnte. Der Eingang wurde von nur wenigen Soldaten bewacht. Dennoch ist in den Quellen nichts über Fluchtversuche enthalten. Die Gefangenen mußten keine harte Arbeit leisten, konnten lesen oder Sport treiben. Es wurden keine schweren Disziplinarstrafen verhängt, etwa Erschießungen, Folterungen oder erschwerte Haftbedingungen. In der Zeit von 1940 bis zur Auflösung des Lagers Anfang 1946 gab es in Miranda nur fünf Todesfälle, durchweg Fälle nicht oder zu spät behandelter Blinddarm- bzw. Lungenentzündungen. ${ }^{12}$

Dennoch war der Aufenthalt für die internierten Personen eine bittere Erfahrung. Ernährung, Wohnverhältnisse und Hygiene waren unerträglich. In einem Barackenraum lebten 70 bis 80 Personen. Die Baracken waren kaum beleuchtet und im Winter schlecht beheizt. Für zeitweilig etwa 4.000 Lagerinsassen gab es nur 18 Duschen, 36 Toiletten und einen einzigen Wasserhahn mit Trinkwasser. Die ständig überbelegte Krankenstation verfügte nur über 30 Betten. Die Ernährung war mangelhaft, allerdings auch nicht schlechter als die der Wachmannschaften. Die Gefangenen konnten Post und stundenweise auch Besuch empfangen, wobei zu bemerken ist, daß die Anreise in diese Lager wegen ihrer Abgelegenheit und der Verkehrsverhältnisse recht mühsam war. Das schlimmste am Lager war die zeitweilige Überbelegung. Ursprünglich für etwa 1.500 Häftlinge geplant, wurden zeitweilig 4.000 bis 5.000 Gefangene festgehalten. Besondere Schwierigkeiten bereiteten die Reibereien zwischen den Nationalitäten, so zwischen jüdischen Insassen unterschiedlicher Herkunft und Polen. Bedrückend war vor allem die völlige Ungewißheit über die Dauer des Lageraufenthalts. Der kürzeste nachweisbare dauerte fünf Monate, der längste über zwei Jahre. ${ }^{13}$

Das andere Lager - Nanclares de la Oca - war kleiner und beherbergte nur etwa 100 Insassen. Die Lebensbedingungen waren ungleich härter, und es diente daher als Disziplinierungsanstalt für unbequeme Häftlinge aus Miranda. Gegen Ende des Krieges wurden vorwiegend Deserteure der deutschen Wehrmacht, die über die Pyrenäen nach Spanien geflohen waren, von Miranda

\footnotetext{
${ }^{12}$ Zahlreiche Materialien über Miranda und Nanclares de la Oca befinden sich in folgenden Archivalien des spanischen Außenministeriums: Ministerio de Asuntos Exteriores (MAE), Madrid: Leg. R 2179: Refugiados Apátridas, exp. 40-44; Leg. R 2182: Extranjeros en el Campo de Nanclares de la Oca, exp. 5-8.

${ }^{13}$ Ysart 1973, 52; Wischnitzer 1956, 181f.; Bachner 1985, 210-212.
} 
dorthin verlegt, weil es in Miranda zwischen ihnen und den anderen Häftlingen zu schweren Konflikten gekommen war. Beide Lager bestanden bis 1948.

Nach Intervention der westalliierten Botschaften und internationaler Hilfsorganisationen verbesserten die spanischen Stellen die Verhältnisse und entspannten die Lage dadurch, daß sie zahlreiche Häftlinge in residencia forzada entließen. Diese Einrichtung, die man schon frühzeitig für Frauen und Kinder eingeführt hatte, gewährte den Emigranten eine relative Freiheit. Sie mußten sich an einem Ort aufhalten und durften ihn ohne Genehmigung nicht verlassen, unterlagen aber sonst keinen weiteren Beschränkungen. Sie lebten in der Regel in Pensionen oder Hotels und wurden von ausländischen Hilfsorganisationen oder durch britische oder amerikanische Gelder ernährt. Dies war nun schon ein großer Fortschritt gegenüber der Inhaftierung in Konzentrationslagern. Es wird geschätzt, daß sich Anfang 1943 unter Einschluß der Lagerhäftlinge zwischen 10.000 und 17.000 Ausländer in Spanien aufhielten. Manche Schätzungen bewegen sich sogar bei 25.000 Ausländern. In der Mehrheit handelte es sich um Franzosen. Etwa 3.400 werden als apátridas bezeichnet, in der Regel ein Synonym für staatenlose jüdische Flüchtlinge aus Mitteleuropa.

Die meisten aus den Lagern entlassenen Flüchtlinge versuchten, so schnell wie möglich Spanien wieder zu verlassen - direkt nach Übersee, nach Palästina oder nach Portugal und von dort weiter. Nur wenige blieben in Spanien in der Regel unfreiwillig, weil sie keine gültigen Papiere für andere Aufnahmeländer besaßen. Sie konnten erst nach Kriegsende Spanien verlassen. Einige wenige Familien schlugen freilich Wurzeln, erhielten eine Arbeitserlaubnis und Anstellung und zogen erst einige Jahre nach Kriegsende weiter. Einen Sonderfall bildete der Übersetzer, Dramaturg und Bühnenschriftsteller Hans Rothe (1894-1977), der schon 1939 aus dem italienischen Exil ins spanische übergewechselt war. Trotz der spanischen Zensur und der Mißgunst der deutschen Botschaft konnte er seine Arbeit in Madrid fortsetzen und sogar zwei seiner Stücke - Ankunft bei Nacht und Huellas borradas (Verwischte Spuren) inszenieren. Er wurde nach Kriegsende von den Alliierten mit der Auflösung der deutschen Kulturinstitute in Spanien beauftragt, bis auch er 1947 das Land wieder verließ.

Von einzelnen Ausnahmen abgesehen, blieben in Spanien keine Emigranten. Die diktatorischen Verhältnisse, die Präsenz und Umtriebe geflüchteter deutscher Nazis, die wirtschaftlichen Schwierigkeiten und die allgemeine Armut boten nichts, was die Emigranten dort hätte halten können. Spanien war für die meisten nur ein Transitland, kein Refugium gewesen, ihr Aufenthalt bedeutete für sie nur eine unfreiwillige Unterbrechung auf der Weiterreise; und so sind auch kaum Spuren geblieben, die auf die Flüchtlingsströme hindeuten, für die Spanien vorübergehend zum Schicksal geworden war. 


\section{Bibliographie}

Abusch, Alexander. 1981. Der Deckname. Memoiren. Berlin/Ost: Dietz.

Bachner, Rudolph. 1985. Flucht ohne Ziel. Ein Leben in der Emigration. Hamburg: Christians.

Bernecker, Walther L. 1983. Spaniens Geschichte seit dem Bürgerkrieg. München: Beck.

Brigada Internacional ist unser Ehrennahme. Erlebnisse ehemaliger Spanienkämpfer. 1976. 2 Bde., ausgew. und eingel. von Hans Maaßen. Frankfurt a. M.: Röderberg.

Detwiler, Ronald. 1962. Hitler, Franco und Gibraltar. Die Frage des spanischen Kriegseintritts in den zweiten Weltkrieg. Wiesbaden: Steiner.

Feuchtwanger, Lion. o.J. Der Teufel in Frankreich. Ein Erlebnisbericht. Mit einem Vorwort von Marta Feuchtwanger. München/Wien: Langen-Müller.

Fischer, Ruth; Arkadaj Maslow. 1990. Abtrünnig wider Willen. Aus Briefen und Manuskripten des Exils. Hg. von Peter Lübbe. München: Oldenbourg.

Fry, Varian. 1986. Auslieferung auf Verlangen. Die Rettung deutscher Emigranten in Marseille 1940/41. Hg. und mit einem Anhang versehen von Wolfgang D. Elfe und Jan Hans. München/Wien: Hanser.

Gilbert, Martin. 1969. Jewish History Atlas. London: Weidenfeld \& Nicolson.

Hamilton, Thomas J. 1943. Appeasement's Child. The Franco Regime in Spain. London: Gollancz.

Hermsdorf, Klaus; Hugo Fetting; Silvia Schlenstedt. 1981. Exil in den Niederlanden und in Spanien (Kunst und Literatur im antifaschistischen Exil 19331945, Bd. 6). Frankfurt am Main: Röderberg.

Hillgruber, Andreas. 1965. Hitlers Strategie: Politik und Kriegführung 19401941. Frankfurt a. M.: Bernhard \& Graefe.

Hoare, Sir Samuel. 1946. Ambassador On Special Mission. London: Collins.

Kerbs, Diethard. 1986. Spanisches Tagebuch. Hans Namuth - Georg Reisner. Berlin: Nishen.

Lustiger, Arno. 1989. Schalom Libertad!: Juden im spanischen Bürgerkrieg. Frankfurt a. M.: Athenäum.

Mühlen, Patrik von zur. 1992. Fluchtweg Spanien - Portugal. Die deutsche Emigration und der Exodus aus Europa 1933-1945. Bonn: J.H.W. Dietz Nachf.

-. 1985. Spanien war ihre Hoffnung. Die deutsche Linke im Spanischen Bürgerkrieg 1936-1939. Bonn: J.H.W. Dietz Nachf. 2. Auf1. 
Robinson, Nehemiah. 1953. Spain of Franco and Its Policies Towards the Jews. New York: Inst. of Jewish Affairs.

Ruhl, Klaus-Jörg. 1975. Spanien im Zweiten Weltkrieg. Franco, die Falange und das Dritte Reich. Hamburg: Hoffmann \& Campe.

Stampfer, Friedrich. 1957. Erfahrungen und Erkenntnisse. Aufzeichnungen aus meinem Leben. Köln: Verlag für Politik und Wirtschaft.

Tartakower, Arieh; Kurt R. Grossmann. 1944. The Jewish Refugee. New York: Inst. of Jewish Affairs.

Wasserstein, Bernard. 1979. Britain and the Jews of Europe, 1939-1945. London: Inst. of Jewish Affairs/Oxford: Clarendon Press.

Wischnitzer, Mark. 1956. Visas to Freedom: The History of the HIAS. Cleveland/New York: World Publisher.

Ysart, Federico. 1973. España y los judios en la Segunda Guerra Mundial. Barcelona: DOPESA. 